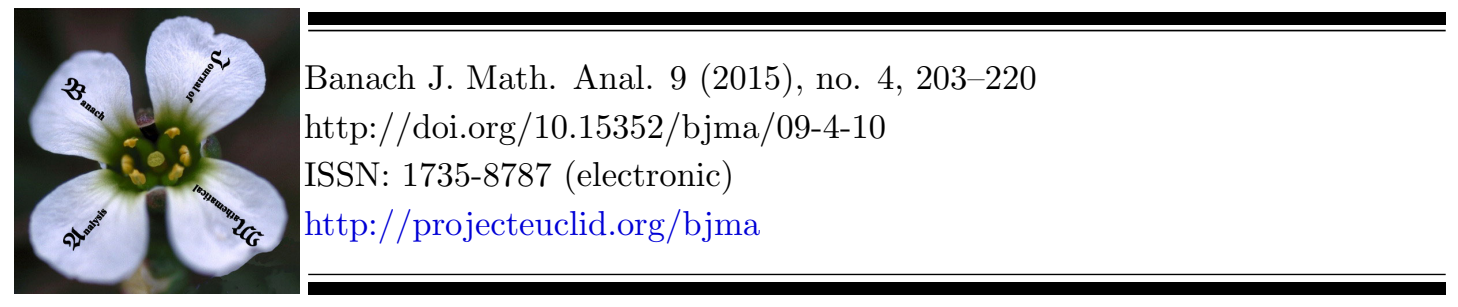

\title{
DIAMETER TWO PROPERTIES IN JAMES SPACES
}

\author{
JULIO BECERRA GUERRERO, GINÉS LÓPEZ-PÉREZ* AND ABRAHAM RUEDA \\ ZOCA
}

Communicated by D. Werner

\begin{abstract}
We study the diameter two properties in the (James type) Banach spaces $J H, J T_{\infty}$ and $J H_{\infty}$. We show that the dual spaces of these three Banach spaces fail every diameter two property. Also, we prove that $J H$ and $J H_{\infty}$ satisfy the strong diameter two property, and so the dual norms of these spaces are octahedral. In addition, we find a closed hyperplane $M$ of $J H_{\infty}$ such that its dual space, $M^{*}$, satisfies the $w^{*}$-strong diameter two property. Finally, we get that the natural norms of $M$ and $M^{*}$ are octahedral.
\end{abstract}

\section{INTRODUCTION AND PRELIMINARIES}

We say that a Banach space has the slice diameter two property (slice-D2P), respectively diameter two property (D2P), strong diameter two property (SD2P), if every slice, respectively non-empty weakly open set, convex combination of slices, in its unit ball has diameter two. We also define the weak-star versions of the above properties: the weak-star slice diameter two property $\left(w^{*}\right.$-slice $\left.\mathrm{D} 2 \mathrm{P}\right)$, the weak-star diameter two property $\left(w^{*}\right.$-D2P) and the weak-star strong diameter two property $\left(w^{*}\right.$-SD2P), asking for the above conditions for $w^{*}$-slices, nonempty relatively $w^{*}$-open subsets and convex combination of $w^{*}$-slices.

The diameter two properties are extremely opposite to the well known RadonNikodym property (RNP) in Banach spaces, since it is well known that the RNP for a Banach space is characterized by the existence of slices with diameter arbitrarily small in every nonempty and bounded subset of the space. Let us remark

Date: Received: Oct. 15, 2014; Revised: Nov. 18, 2014; Accepted: Jan. 6, 2015.

* Corresponding author.

2010 Mathematics Subject Classification. Primary 46B20; Secondary 46B22, 52A10.

Key words and phrases. James space, diameter two properties, octahedral norm. 
that RNP is an isomorphic property, that is, independent on the equivalent norm considered, while the diameter two properties are purely geometric properties, depending on the equivalent norm considered.

In the last years a wide class of Banach spaces satisfying some of the diameter two properties have appeared, as infinite-dimensional uniform algebras [14], infinite-dimensional $C^{*}$-algebras [1], non-reflexive $M$-embedded spaces [9], Banach spaces with the Daugavet property [16], etc. However, the diameter two properties have not so far been much investigated for what one would describe as non- classical Banach spaces.

Probably the origin of non-classical Banach spaces is [10], where the space $J$ (James space) is constructed in order to provide an example of a non-reflexive Banach space which fails to contain an isomorphic copy of $c_{0}$ or $\ell_{1}$. A year later [11], James went further and modified the definition of the norm in order to show that $J$ and $J^{* *}$ are isometrically isomorphic despite the fact that $\mathrm{J}$ is non-reflexive (see [5] or [13] for background on the space $J$ ). It is known that $J$ and $J^{*}$ have the RNP both being separable duals.

After the construction of $J$, James defined in [12] the space $J T$ (James tree space), exhibiting an example of a separable Banach space whose dual space is non-separable and so that it does not contain any isomorphic copy of $\ell_{1}$, giving a negative answer to a conjecture of Stephan Banach (again we refer to [5] or [13] for background on JT space). It is known that JT satisfies the RNP and $B$, the predual of $J T$, fails the RNP [5]. However, $B$ is far from satisfying the slice diameter two property. Indeed, in [15, Theorem 5.1] it is proved the existence of a constant $0<\beta<2$ such that every closed and convex subset of the unit ball of $B$ has a slice whose diameter is at most $\beta$ (in fact, it is conjectured in $[15$, Remark 5.2] that the above constant $\beta$ could be, at most, $\sqrt{2}$ ). In any case, $B$ can be considered as the first non-classical Banach space where the size of slices is studied.

Motivated by the analysis of $B$, the aim of this note is to study the slices of the unit ball for some related, non-classical Banach spaces. Indeed, in section 2 we focus on the $J T_{\infty}^{*}$ space, by showing that $J T_{\infty}^{*}$ fails the $w^{*}$-slice diameter two property, and so every diameter two property. Then the space $B_{\infty}$, the predual space of $J T_{\infty}$, fails every diameter two property. In fact, we prove that the inf of the diameters of slices in the unit ball of $B_{\infty}$ is, at most, $\sqrt{2}$. The same fact also holds for the unit ball of the predual space $B$ of $J T$, and so we can confirm [15, Remark 5.2].

In section 3 we prove that the unit ball of $J H$ has Fréchet differentiability points and, as a consequence, the unit ball of $J H^{*}$ contains $w^{*}$-slices of arbitrarily small diameter. Also, it is proved in this section that $J H$ has the strong diameter two property. As a consequence, we get that the norm in the dual space $J H^{*}$ is octahedral.

In section 4 we introduce the $J H_{\infty}$ space, a Banach space which is not linearly isomorphic to $J H$, since we show that unit ball of $J H_{\infty}^{*}$ has $w^{*}$ slices of diameter strictly less than 2 and so $J H \infty^{*}$ fails every diameter two property. Moreover, 
it is proved that $J H_{\infty}$ has the strong diameter two property, and so we deduce from this fact that the norm of $J H_{\infty}^{*}$ is octahedral.

Finally, in section 5, we find a closed hyperplane $M$ of $J H_{\infty}$ such that $M^{*}$ satisfies the $w^{*}$-strong diameter two property and we deduce that the natural norms of $M$ and $M^{*}$ are octahedral.

We shall now introduce some notation. We will consider real Banach spaces. $B_{X}$, respectively $S_{X}$, stands for the closed unit ball, respectively the unit sphere, of the Banach space $X$. We denote by $X^{*}$ the topological dual space of $X$. A slice of a bounded subset $A \subseteq X$ is a set defined by

$$
S\left(A, x^{*}, \alpha\right):=\left\{x \in A \mid x^{*}(x)>\sup x(A)-\alpha\right\}
$$

whenever $x^{*} \in X^{*}$ and $0<\alpha$. Similarly, a $w^{*}$-slice of a bounded subset $B \subseteq X^{*}$ is a set given by

$$
S(B, x, \alpha):=\left\{x^{*} \in B \mid x^{*}(x)>\sup x(B)-\alpha\right\}
$$

whenever $x \in X$ and $0<\alpha$.

Recall that the norm of a Banach space $X$ is octahedral (see [4]) if for every $\varepsilon>0$ and for every finite-dimensional subspace $Y$ of $X$ there is $x \in S_{X}$ such that

$$
\|\lambda x+y\|>(1-\varepsilon)(|\lambda|+\|y\|)
$$

for every $y \in Y$ and for every scalar $\lambda$. We remark that the norm of a Banach space $X$ is octahedral if, and only if, $X^{*}$ satisfies the $w^{*}$-strong diameter two property and, dually, the norm of $X^{*}$ is octahedral if, and only if, $X$ satisfies the strong diameter two property (see [2]).

Also we recall that a Banach space $X$ has the Daugavet property if the equation

$$
\|T+I\|=1+\|T\|
$$

holds for every rank one, linear and bounded operator on $X$, where $I$ denotes the identity operator.

The Banach space $X$ is said to have the almost Daugavet property if there is some norming subspace $Y$ of $X^{*}$ such that the equation (1.1) holds for every rank one operator $T$ given by $T=x \otimes y^{*}$ for $x \in X$ and $y^{*} \in Y$. It is known [8] that, for a separable Banach space, having octahedral norm and satisfying the almost Daugavet property are equivalent properties. The above two equivalent properties are also equivalent to the fact that $X^{*}$ has the $w^{*}$-strong diameter two property, as can be deduced from the comments in the above paragraph. These facts will be used freely below.

The following known result, see Lemma 2.1 and Proposition 3.1 in [3], will be useful in order to estimate the infimum of diameters of $w^{*}$ - slices in dual spaces.

Theorem 1.1. Let $X$ be a Banach space and assume that $A \subseteq X^{*}$ satisfies $B_{X^{*}}=\overline{c o}^{w^{*}}(A)$. If $x \in S_{X}$, then

$$
\inf _{\alpha>0} \operatorname{diam}(S(A, x, \alpha))=\inf _{\alpha>0} \operatorname{diam}\left(S\left(B_{X^{*}}, x, \alpha\right)\right) .
$$




\section{THE SPACE $J T_{\infty}$.}

We begin with the construction of $J T_{\infty}$. Let us define

$$
T:=\left\{\left(\alpha_{1}, \ldots, \alpha_{k}\right) \mid k \in \mathbb{N}, \alpha_{1}, \ldots, \alpha_{n} \in \mathbb{N}\right\} \cup\{\emptyset\} .
$$

Given $\left(\alpha_{1}, \ldots, \alpha_{k}\right),\left(\beta_{1}, \ldots, \beta_{p}\right) \in T$ we say that

$$
\left(\alpha_{1}, \ldots, \alpha_{k}\right) \leq\left(\beta_{1}, \ldots, \beta_{p}\right) \Leftrightarrow\left\{\begin{array}{c}
\left|\left(\alpha_{1}, \ldots, \alpha_{k}\right)\right| \leq\left|\left(\beta_{1}, \ldots, \beta_{p}\right)\right| \\
\alpha_{i}=\beta_{i}
\end{array} \forall 1 \leq i \leq k,\right.
$$

where $\left|\left(\alpha_{1}, \ldots, \alpha_{n}\right)\right|:=n$ and $|\emptyset|:=0$. This binary relation defines a partial order on $T$.

A segment in $T$ is a totally ordered and finite subset $S \subseteq T$.

Given $x: T \longrightarrow \mathbb{R}$, let us consider

$$
\|x\|=\sup \left(\sum_{i=1}^{n}\left(\sum_{t \in S_{i}} x(t)\right)^{2}\right)^{\frac{1}{2}},
$$

where the sup is taken over all families $\left\{S_{1}, \ldots, S_{n}\right\}$ of disjoint segments of $T$.

Now $J T_{\infty}$ is defined as the completion of the space of finitely nonzero functions defined on $T$ (i.e. functions $x: T \longrightarrow \mathbb{R}$ such that $\{t \in T \mid x(t) \neq 0\}$ is finite) for the above norm. Given $\alpha \in T$ let us define

$$
e_{\alpha}(\beta):= \begin{cases}1 & \text { if } \beta=\alpha \\ 0 & \text { otherwise. }\end{cases}
$$

Then it is known that $\left\{e_{\alpha}\right\}_{\alpha \in T}$ is a Schauder basis for $J T_{\infty}$ and that $J T_{\infty}$ is a dual space. We denote by $\left\{e_{\alpha}^{*}\right\}_{\alpha \in T}$ the biorthogonal sequence of $\left\{e_{\alpha}\right\}_{\alpha \in T}$. Then $B_{\infty}:=\operatorname{span}\left\{e_{\alpha}^{*} / \alpha \in T\right\}$, where the closure is taken in $J T_{\infty}^{*}$, is a complete predual of $J T_{\infty}$.

The space $J T_{\infty}$ was introduced in [6], where it is proved that $B_{\infty}$ fails the Radon-Nikodym property. Furthermore, every infinite-dimensional subspace of $J T_{\infty}$ contains an isomorphic copy of $\ell_{2}$ and so $J T_{\infty}$ does not contain isomorphic copies of $\ell_{1}$.

Now we pass to study the size of slices in $B_{J T_{\infty}^{*}}$. As in [15], we define a molecule as a functional of the form

$$
x^{*}:=\sum_{i=1}^{n} \lambda_{i} f_{S_{i}}
$$

for $S_{1}, \ldots, S_{n}$ disjoint segments of $T$ and $\sum_{i=1}^{n} \lambda_{i}^{2} \leq 1$, where $f_{S} \in B_{J T_{\infty}^{*}}$ is defined by the equation

$$
f_{S}(x):=\sum_{t \in S} x(t)
$$

whenever $S \subseteq T$ is a segment of $T$.

Denote by $M$ the set of molecules in $J T_{\infty}^{*}$ and note that $M$ is a symmetric subset of $B_{J T_{\infty}^{*}}$. The following lemma states that $M$ is in fact a norming subset of $B_{J T_{\infty}^{*}}$. 
Lemma 2.1. $M$ is a norming subset of $B_{J T_{\infty}^{*}}$. As a consequence

$$
B_{J T_{\infty}^{*}}=\overline{c o}^{w^{*}}(M) .
$$

Proof. Let $x \in S_{J T_{\infty}}$ be a finitely nonzero function defined on $T$. Pick an arbitrary $0<\varepsilon<1$ and take $0<\delta<1$ such that $(1-\delta)^{2}>1-\varepsilon$. By the definition of the norm in $J T_{\infty}$ we deduce that there exist $S_{1}, \ldots, S_{n}$ disjoint segments in $T$ such that

$$
\left(\sum_{i=1}^{n} f_{S_{i}}(x)^{2}\right)^{\frac{1}{2}}>1-\delta .
$$

For every $i \in\{1, \ldots, n\}$ we define $\lambda_{i}:=f_{S_{i}}(x)$ and we note that, from the definition of the norm in $J T_{\infty}, \sum_{i=1}^{n} \lambda_{i}^{2} \leq 1$. Moreover, in view of the last inequality, we have

$$
\sum_{i=1}^{n} \lambda_{i} f_{S_{i}}(x)=\sum_{i=1}^{n} f_{S_{i}}(x)^{2}>(1-\delta)^{2}>1-\varepsilon .
$$

As a consequence we can find elements in $M$ whose evaluation at $x$ is as close to $\|x\|$ as desired. Hence $M$ is a norming subset of $B_{J H_{\infty}^{*}}$.

From a separation argument we get now that $B_{J T_{\infty}^{*}}=\overline{C O}^{w^{*}}(M)$.

Using the previous lemma, we will prove that there exist $w^{*}$-slices in $B_{J T_{\infty}^{*}}$ with diameter strictly less than 2 .

Theorem 2.2. There exists $x \in S_{J T_{\infty}}$ such that

$$
\inf _{\alpha>0} \operatorname{diam} S\left(B_{J T_{\infty}^{*}}, x, \alpha\right) \leq \sqrt{2} .
$$

Proof. Let $0<\varepsilon<1 / 2$. Pick $0<\delta<\min \{\varepsilon, 2 \varepsilon(1-\varepsilon)\}$ and $0<\alpha<1 / 2$ such that $(1-\alpha)^{2}>1-\delta$. Let us define

$$
x:=(1-\varepsilon) e_{\emptyset}+\varepsilon e_{(1)} \in S_{J T_{\infty}} .
$$

We consider $S:=S(M, x, \alpha)$. Take $\sum_{i=1}^{n} \lambda_{i} f_{S_{i}}, \sum_{j=1}^{m} \mu_{j} f_{T_{j}} \in S$.

In view of the form of $x$ we can assure the existence of $i \in\{1, \ldots, n\}, j \in$ $\{1, \ldots, m\}$ such that $\{\emptyset,(1)\} \subseteq S_{i} \cap T_{j}$. Indeed, it is clear that $\left(\cup_{i=1}^{n} S_{i}\right) \cap\{\emptyset,(1)\} \neq$ $\emptyset$, since $\sum_{i=1}^{n} \lambda_{i} f_{S_{i}} \in S$. Now it is not possible that $\left(\cup_{i=1}^{n} S_{i}\right) \cap\{\emptyset,(1)\}=\{(\emptyset)\}$ nor $\left(\cup_{i=1}^{n} S_{i}\right) \cap\{\emptyset,(1)\}=\{(1)\}$, since $0<\varepsilon<1 / 2,0<\alpha<1 / 2, \sum_{i=1}^{n} \lambda_{i}^{2} \leq 1$ and $\sum_{i=1}^{n} \lambda_{i} f_{S_{i}} \in S$. Finally, it is not possible that there exist $i \neq j$ such that $\{(\emptyset)\} \in S_{i}$ and $\{(1)\} \in S_{j}$, since if this is the case, we have that $(1-\varepsilon) \lambda_{i}+\varepsilon \lambda_{j}>$ $1-\alpha$. Hence

$$
(1-\alpha)^{2}<\left((1-\varepsilon)^{2}+\varepsilon^{2}\right)\left(\lambda_{i}^{2}+\lambda_{j}^{2}\right) \leq(1-\varepsilon)^{2}+\varepsilon^{2}
$$

and thus, using the conditions on $\alpha, \delta$ and $\varepsilon$, we get

$$
1-2(\varepsilon(1-\varepsilon))<1-\delta<(1-\varepsilon)^{2}+\varepsilon^{2},
$$

which is a contradiction. This proves the existence of $i$ such that $\{\emptyset,(1)\} \subseteq S_{i}$. The same argument proves the existence of $j$ such that $\{\emptyset,(1)\} \subseteq T_{j}$. Of course, 
we assume without loss of generality that $i=j=1$. Now

$$
\sum_{i=1}^{n} \lambda_{i} f_{S_{i}}(x)=\lambda_{1}(1-\varepsilon+\varepsilon)=\lambda_{1}>1-\alpha \Rightarrow \lambda_{1}^{2}>(1-\alpha)^{2}>1-\delta .
$$

From $\sum_{i=1}^{n} \lambda_{i}^{2} \leq 1$ we get $\sum_{i=2}^{n} \lambda_{i}^{2}<\delta$. By a similar argument $\mu_{1}^{2}>1-\delta$ and hence $\sum_{j=2}^{m} \mu_{j}^{2}<\delta$.

In order to estimate $\left\|\sum_{i=1}^{n} \lambda_{i} f_{S_{i}}-\sum_{j=1}^{m} \mu_{j} f_{T_{j}}\right\|$ pick $y \in S_{J T_{\infty}}$. Hence

$$
\begin{gathered}
\left|\left(\sum_{i=1}^{n} \lambda_{i} f_{S_{i}}-\sum_{j=1}^{m} \mu_{j} f_{T_{j}}\right)(y)\right| \leq \underbrace{\left|\lambda_{1} f_{S_{1}}(y)-\mu_{1} f_{T_{1}}(y)\right|}_{\mathrm{A}}+ \\
\underbrace{\left|\sum_{i=2}^{n} \lambda_{i} f_{S_{i}}(y)-\sum_{j=2}^{m} \mu_{j} f_{T_{j}}(y)\right|}_{\mathrm{B}} .
\end{gathered}
$$

We shall begin by estimating $B$. In view of Hölder's inequality we have

$$
\begin{gathered}
B \leq \sum_{i=2}^{n}\left|\lambda_{i}\right|\left|f_{S_{i}}(y)\right|+\sum_{j=2}^{m}\left|\mu_{j}\right|\left|f_{T_{j}}(y)\right| \leq \\
\leq\left(\sum_{i=2}^{n} \lambda_{i}^{2}+\sum_{j=2}^{m} \mu_{j}^{2}\right)^{\frac{1}{2}}\left(\sum_{i=2}^{n} f_{S_{i}}(y)^{2}+\sum_{j=2}^{m} f_{T_{j}}(y)^{2}\right)^{\frac{1}{2}} \leq(2 \delta)^{\frac{1}{2}} 2^{\frac{1}{2}}=2 \sqrt{\delta}
\end{gathered}
$$

because $\sum_{i=2}^{n} f_{S_{i}}(y)^{2} \leq\|y\|^{2}=1, \sum_{j=2}^{m} f_{T_{j}}(y)^{2} \leq 1$ due to the disjointness of $\left\{S_{2}, \ldots, S_{n}\right\}$ and $\left\{T_{2}, \ldots, T_{m}\right\}$. So $B \leq 2 \sqrt{\delta}$. Now we will estimate $A$.

$$
A \leq\left|\lambda_{1}-\mu_{1}\right|\left|f_{T_{1} \cap S_{1}}(y)\right|+\left|\lambda_{1}\right|\left|f_{S_{1} \backslash T_{1}}(y)\right|+\left|\mu_{1}\right|\left|f_{T_{1} \backslash S_{1}}(y)\right| .
$$

From $1 \geq \lambda_{1}>1-\alpha$ and $1 \geq \mu_{1}>1-\alpha$ we get $\left|\lambda_{1}-\mu_{1}\right|<\alpha$. Hence

$$
\begin{gathered}
A \leq \alpha\left\|f_{T_{1} \cap S_{1}}|\|y\|+| \lambda_{1}|| f_{S_{1} \backslash T_{1}}(y)|+| \mu_{1}|| f_{T_{1} \backslash S_{1}}(y) \mid\right. \\
=\alpha+\left|\lambda_{1}\right|\left|f_{T_{1} \backslash S_{1}}(y)\right|+\left|\mu_{1}\right|\left|f_{T_{1} \backslash S_{1}}(y)\right| \leq \alpha+\left|f_{S_{1} \backslash T_{1}}(y)\right|+\left|f_{T_{1} \backslash S_{1}}(y)\right| .
\end{gathered}
$$

Again, applying Hölder's inequality, we have

$$
A \leq \alpha+\sqrt{2}\left(f_{S_{1} \backslash T_{1}}(y)^{2}+f_{T_{1} \backslash S_{1}}(y)^{2}\right)^{\frac{1}{2}} .
$$

Since $\left\{S_{1} \backslash T_{1}, T_{1} \backslash S_{1}\right\} \subseteq T$ is a family of disjoint segments, we have that $f_{S_{1} \backslash T_{1}}(y)^{2}+f_{T_{1} \backslash S_{1}}(y)^{2} \leq\|y\|^{2}=1$. Hence

$$
A \leq \alpha+\sqrt{2} .
$$

Summarizing gives

$$
\left|\left(\sum_{i=1}^{n} \lambda_{i} f_{S_{i}}-\sum_{j=1}^{m} \mu_{j} f_{T_{j}}\right)(y)\right| \leq \alpha+\sqrt{2}+2 \sqrt{\delta} .
$$


From the arbitrariness of $y \in S_{J T_{\infty}}$ we have that

$$
\begin{aligned}
\left\|\sum_{i=1}^{n} \lambda_{i} f_{S_{i}}-\sum_{j=1}^{m} \mu_{j} f_{T_{j}}\right\| & =\sup _{y \in S_{J T_{\infty}}}\left|\left(\sum_{i=1}^{n} \lambda_{i} f_{S_{i}}-\sum_{j=1}^{m} \mu_{j} f_{T_{j}}\right)(y)\right| \\
& \leq \sqrt{2}+\alpha+2 \sqrt{\delta} .
\end{aligned}
$$

Hence

$$
\operatorname{diam}(S) \leq \sqrt{2}+\alpha+2 \sqrt{\delta} .
$$

So

$$
\inf _{\alpha>0} \operatorname{diam}(S(M, x, \alpha)) \leq \sqrt{2}+2 \sqrt{\delta} .
$$

Since $0<\delta<\varepsilon$ is arbitrary, we deduce that

$$
\inf _{\alpha>0} \operatorname{diam}(S(M, x, \alpha)) \leq \sqrt{2} .
$$

In view of Lemma 2.1, Theorem 1.1 applies and

$$
\inf _{\alpha>0} \operatorname{diam}\left(S\left(B_{J T_{\infty}^{*}}, x, \alpha\right)\right) \leq \sqrt{2},
$$

and we are done.

In view of Theorem 2.2, for each $0<\varepsilon<2-\sqrt{2}$ we can find a $w^{*}$-slice $S$ in $B_{J T_{\infty}^{*}}$ such that $\operatorname{diam}(S)<\sqrt{2}+\varepsilon$. In particular, $J T_{\infty}^{*}$ fails the $w^{*}$-slice diameter two property and hence $B_{\infty}$ fails every diameter two property, since the inf of the diameters of slices in the unit ball of $B_{\infty}$ agrees with the inf of the diameters of $w^{*}$-slices in the unit ball of $J T_{\infty}^{*}$. In fact, this inf is, at most, $\sqrt{2}$. Furthermore, it is possible to obtain the same result for the space $B$, the predual of $J T$, with the above proof, which shows that the conjecture in [15], that the inf of diameters of slices in the unit ball in $B$ is at most $\sqrt{2}$ holds.

\section{The SPACE $J H$.}

We begin with the construction of $J H$. Following [5] we denote by

$$
T:=\left\{(n, i) / 0 \leq n<\infty, 0 \leq i<2^{n}\right\}
$$

the dyadic tree. We say that $(n+1,2 i)$ and $(n+1,2 i+1)$ are offsprings of $(n, i)$ for every $(n, i) \in T$. A segment will be a non-empty finite sequence

$$
S=\left\{t_{1}, \ldots, t_{n}\right\}
$$

such that $t_{j+1}$ is an offspring of $t_{j}$ for every $j \in\{1, \ldots, n-1\}$.

Now we are ready to define a partial order in $T$ : Given $t_{1}, t_{2} \in T$ we say that $t_{1}<t_{2}$ if, and only if, $t_{1} \neq t_{2}$ and there exists a segment such that $t_{1}$ is the first element of the segment and $t_{2}$ is the last one of it.

The set

$$
\left\{(n, i) \mid 0 \leq i<2^{n}\right\}
$$

is called the $n$-th level of $T$ for every $0 \leq n<\infty$. Given $a \in T$, lev $(a)$ is defined as the integer number such that $a$ belongs to the $\operatorname{lev}(a)$-th level.

Given $n, m \in \mathbb{N}, n \leq m$ we will say that a subset $S \subseteq T$ is an $n-m$ segment if 
- For every $n \leq k \leq m$ there exists only one element in $S$ which is in the $k$-th level of $T$,

- If $(p, i),(q, j) \in S$ and $p<q$ then $(p, i)<(q, j)$ (in other words, $S$ is a totally ordered subset of $T$ ).

Given $x: T \longrightarrow \mathbb{R}$ and $S \subseteq T$ a segment in $T$, we define

$$
f_{S}(x):=\sum_{t \in S} x(t) \text {. }
$$

Note that the above sum is well defined because $S$ is finite. if:

Given $\left\{S_{1}, \ldots, S_{n}\right\}$ a family of segments in $T$ we say that they are admissible

i) There exist $p \leq q$ natural numbers such that $S_{i}$ is a $p-q$ segment for every $i \in\{1, \ldots, n\}$.

ii) $S_{i} \cap S_{j}=\emptyset$ whenever $i \neq j$.

Given $x: T \longrightarrow \mathbb{R}$, a finitely nonzero function, we define

$$
\|x\|:=\max \sum_{i=1}^{n}\left|f_{S_{i}}(x)\right|=\max \sum_{i=1}^{n}\left|\sum_{t \in S_{i}} x(t)\right|,
$$

where the maximum is taken over all families $S_{1}, \ldots, S_{n}$ of admissible segments in $T$.

Now define $J H$ as the completion of the space of finitely nonzero functions on $T$ in the above norm.

Given $t \in T$ we define $e_{t} \in J H$ by the equation

$$
e_{t}(s):=\left\{\begin{array}{cc}
1 & \text { if } t=s \\
0 & \text { otherwise }
\end{array} .\right.
$$

Then it is known that $\left\{e_{t}\right\}_{t \in T}$ defines a Schauder basis in $J H$.

The $J H$ space was introduced by J. Hagler in [7], where it is proved that $J H$ is a separable Banach space such that $J H^{*}$ is not separable and every infinitedimensional subspace of $J H$ contains an isomorphic copy of $c_{0}$. In particular $J H$ contains an isomorphic copy of $c_{0}$, so it can not be a dual space [13, Proposition 2.e.8].

Lemma 3.1. Let $x: T \longrightarrow \mathbb{R}$ be a finitely non-zero function and $n \in \mathbb{N} \backslash\{1\}$ such that

$$
\|x\| \leq 1-\frac{1}{n}
$$

Pick $a \in T$ such that $\operatorname{lev}(a)>\max _{t \in \operatorname{supp}(x)}$ lev $(t)$. Let $\ell \in \mathbb{N}$ big enough such that there exists $t_{1}, \ldots, t_{n} \in T$ so that

- $\operatorname{lev}\left(t_{i}\right)=\ell$ for each $i$.

- $a<t_{i}$ for all $i$.

If we define $y: T \longrightarrow \mathbb{R}$ such that

$$
y(t):=\left\{\begin{array}{cc}
x(t) & \text { when } t \in \operatorname{supp}(x) \\
\mu_{i} \frac{1}{n} & \text { when } t=t_{i} \text { for } i \in\{1, \ldots, n\} \\
0 & \text { otherwise }
\end{array}\right.
$$


where $\mu_{i} \in\{-1,1\}$ for every $i \in\{1, \ldots, n\}$, then $\|y\| \leq 1$.

Proof. Let $\left\{S_{1}, \ldots, S_{k}\right\}$ be a family of admissible segments in $T, \lambda_{1}, \ldots, \lambda_{k} \in$ $\{-1,1\}$ and define

$$
x^{*}:=\sum_{i=1}^{k} \lambda_{i} f_{S_{i}} .
$$

In order to prove that $\|y\| \leq 1$, we have to show that $x^{*}(y) \leq 1$, according to the definition of the norm in $J H$.

We consider the following possibilities:

$\bigcup_{i=1}^{k} S_{i} \cap\left\{t_{1}, \ldots, t_{n}\right\}=\emptyset$

In this case we have, in view of the definition of $y$, that

$$
x^{*}(y)=x^{*}(x) \leq\|x\| \leq 1-\frac{1}{n}
$$

by hypothesis.

(2)

$\bigcup_{i=1}^{k} S_{i} \cap\left\{t_{1}, \ldots, t_{n}\right\} \neq \emptyset$, but $\bigcup_{i=1}^{k} S_{i} \cap \operatorname{supp}(x)=\emptyset$.

In this case we have

$$
x^{*}(y)=\sum_{i=1}^{k} \lambda_{i} f_{S_{i}}(y) \leq \sum_{i=1}^{n} \frac{1}{n}=1
$$

$\bigcup_{i=1}^{k} S_{i} \cap\left\{t_{1}, \ldots, t_{n}\right\} \neq \emptyset$ and $\bigcup_{i=1}^{k} S_{i} \cap \operatorname{supp}(x) \neq \emptyset$

Finally, in this case we have that there exists only one $i \in\{1, \ldots, n\}$ such that $a \in S_{i}$ (otherwise $\bigcup_{i=1}^{k} S_{i} \cap\left\{t_{1}, \ldots, t_{n}\right\}=\emptyset$ in view of the order defined on $T$ ). We can assume, without loss of generality, that $i=1$. If $S_{j}$ is a $p-q$ segment, we can write

$$
S_{j}:=T_{j} \cup R_{j}
$$

where $T_{j}$ is a $p-(\ell-1)$ segment and $R_{j}$ is a $\ell-q$ segment for each $j \in\{1, \ldots, k\}$.

In view of the disjointness of $S_{1}, \ldots, S_{k}$ we have for each $j \in\{2, \ldots, n\}$ that $S_{j} \cap\left\{t_{1}, \ldots, t_{n}\right\}=\emptyset$. In addition, as $\ell>\max _{t \in \operatorname{supp}(x)} l e v(t)$, we deduce that

$$
f_{R_{i}}(y)=0 \forall i \in\{2, \ldots, n\}
$$

Hence

$$
x^{*}(y)=\sum_{i=1}^{k} \lambda_{i} f_{T_{i}}(y)+\lambda_{1} f_{R_{1}}(y) .
$$

Now we have that $\left\{T_{1}, \ldots, T_{k}\right\}$ is a family of admissible segments on $T$. Hence

$$
x^{*}(y) \leq\|x\|+\lambda_{1} f_{R_{1}}(x) \leq 1-\frac{1}{n}+f_{R_{1}}(y) .
$$


Now, as $\left\{t_{1}, \ldots, t_{n}\right\}$ are incomparable nodes on $T$ at the same level, we have that $\left\{t_{1}, \ldots, t_{n}\right\} \cap R_{1}$ has one element. Hence

$$
x^{*}(y) \leq 1-\frac{1}{n}+f_{R_{1}}(y) \leq 1-\frac{1}{n}+\frac{1}{n}=1 .
$$

By the previous discussion we deduce that $\|y\| \leq 1$, as desired.

Theorem 3.2. JH has the strong diameter two property (and so the norm of $J H^{*}$ is octahedral).

Proof. Let $C:=\sum_{i=1}^{n} \lambda_{i} S\left(B_{J H}, x_{i}^{*}, \alpha\right)$ be a convex combination of slices of $B_{J H}$. Let us prove that $\operatorname{diam}(C)=2$.

To this aim pick $x_{i}: T \longrightarrow \mathbb{R}$ a finitely non-zero supported function on $T$ such that $\left\|x_{i}\right\|<1$ and

$$
x_{i}^{*}\left(x_{i}\right)>1-\alpha,
$$

for each $i \in\{1, \ldots, n\}$. For each $i \in\{1, \ldots, n\}$ we can find $a_{i} \in \operatorname{supp}\left(x_{i}\right)$ such that $\operatorname{lev}\left(a_{i}\right)=\max _{t \in \operatorname{supp}\left(x_{i}\right)} \operatorname{lev}(t)$.

As $\left\|x_{i}\right\|<1$ for each $i \in\{1, \ldots, n\}$ we can find $m \in \mathbb{N}$ such that $\left\|x_{i}\right\| \leq 1-\frac{1}{m}$ for each $i \in\{1, \ldots, n\}$. Now we can find $a \in T$ such that $\operatorname{lev}(a)>\max _{1 \leq i \leq n} \operatorname{lev}\left(a_{i}\right)$, $k>\max _{1 \leq i \leq n} \operatorname{lev}\left(a_{i}\right)$ big enough and $\left\{t_{1}^{i}, \ldots, t_{m}^{i}\right\}$ a family of nodes on $T$ at level $k$ such that $a<t_{p}^{i}$ for each $i \in\{1, \ldots, n\}, p \in\{1, \ldots, 2 m\}$ and such that

$$
t_{p}^{i} \neq t_{q}^{j} \text { if } i \neq j \text { or } p \neq q \text {. }
$$

In other words, the last condition guaranties that $\left\{t_{p}^{i} \mid i \in\{1, \ldots, n\}, p \in\right.$ $\{1, \ldots, 2 m\}\}$ is a family of pairwise different nodes at level $k$ which are bigger than $a$.

For each $i \in\{1, \ldots, n\}$ we define $y_{i}, z_{i}: T \longrightarrow \mathbb{R}$ finitely non-zero functions on $T$ as follows

$$
y_{i}(t):=\left\{\begin{array}{ccc}
x_{i}(t) & \text { if } & t \in \operatorname{supp}\left(x_{i}\right) \\
\operatorname{sign}\left(x_{i}^{*}\left(e_{t_{p}^{i}}\right)\right) \frac{1}{m} & \text { if } t=t_{p}^{i}, & \text { for } p \in\{1, \ldots, m\} \\
0 & & \text { otherwise }
\end{array}\right.
$$

and

$$
z_{i}(t):=\left\{\begin{array}{ccc}
x_{i}(t) & \text { if } & t \in \operatorname{supp}\left(x_{i}\right) \\
\operatorname{sign}\left(x_{i}^{*}\left(e_{t_{p}^{i}}\right)\right) \frac{1}{m} & \text { if } t=t_{p}^{i}, & \text { for } p \in\{m+1, \ldots, 2 m\} \\
0 & & \text { otherwise }
\end{array} .\right.
$$

In view of Lemma 3.1 we have that $\left\|y_{i}\right\| \leq 1$ and $\left\|z_{i}\right\| \leq 1$.

Let us prove that, in fact, $y_{i}, z_{i} \in S\left(B_{J H}, x_{i}^{*}, \alpha\right)$ for each $i \in\{1, \ldots, n\}$. To this aim pick $i \in\{1, \ldots, n\}$. We shall prove that $y_{i} \in S\left(B_{J H}, x_{i}^{*}, \alpha\right)$, the case of $z_{i}$ is similar. Using the linearity of $x_{i}^{*}$ we have

$$
x_{i}^{*}\left(y_{i}\right)=x_{i}^{*}\left(x_{i}\right)+\sum_{p=1}^{m} \frac{1}{m} \operatorname{sign}\left(x_{i}^{*}\left(e_{t_{p}^{i}}\right)\right) x_{i}^{*}\left(e_{t_{p}^{i}}\right)=
$$




$$
=x_{i}^{*}\left(x_{i}\right)+\sum_{p=1}^{m} \frac{1}{m}\left|x_{i}^{*}\left(e_{t_{p}^{i}}\right)\right| \geq x_{i}^{*}\left(x_{i}\right)>1-\alpha .
$$

Hence $\sum_{i=1}^{n} \lambda_{i} y_{i}, \sum_{i=1}^{n} \lambda_{i} z_{i} \in C$. Then

$$
\operatorname{diam}(C) \geq\left\|\sum_{i=1}^{n} \lambda_{i} y_{i}-\sum_{i=1}^{n} \lambda_{i} z_{i}\right\|
$$

Now we shall prove that $\left\|\sum_{i=1}^{n} \lambda_{i} y_{i}-\sum_{i=1}^{n} \lambda_{i} z_{i}\right\|=2$. To this aim note that $\left\{\left\{t_{p}^{i}\right\} / i \in\{1, \ldots, n\}, p \in\{1, \ldots, 2 m\}\right\}$ is a family of admissible segments on $T$. Hence

$$
f:=\sum_{i=1}^{n} \sum_{p=1}^{m} \operatorname{sign}\left(x_{i}^{*}\left(e_{t_{p}^{i}}\right)\right) f_{\left\{\left(t_{p}^{i}\right)\right\}}-\sum_{p=m+1}^{2 m} \operatorname{sign}\left(x_{i}^{*}\left(e_{t_{p}^{i}}\right)\right) f_{\left\{\left(t_{p}^{i}\right)\right\}}
$$

is an element on $J H^{*}$ whose norm is, at most, one (in view of the definition of the norm in $J H)$. So

$$
\begin{gathered}
\left\|\sum_{i=1}^{n} \lambda_{i} y_{i}-\sum_{i=1}^{n} \lambda_{i} z_{i}\right\| \geq f\left(\sum_{i=1}^{n} \lambda_{i} y_{i}-\sum_{i=1}^{n} \lambda_{i} z_{i}\right)= \\
=\sum_{i=1}^{n} \lambda_{i} \frac{1}{m} \sum_{p=1}^{m} \operatorname{sign}\left(x_{i}^{*}\left(e_{t_{p}^{i}}\right)\right)^{2}+\lambda_{i} \frac{1}{m} \sum_{p=m+1}^{2 m} \operatorname{sign}\left(x_{i}^{*}\left(e_{t_{p}^{i}}\right)\right)^{2}= \\
=2 \sum_{i=1}^{n} \lambda_{i}=2 .
\end{gathered}
$$

So $\left\|\sum_{i=1}^{n} \lambda_{i} y_{i}-\sum_{i=1}^{n} \lambda_{i} z_{i}\right\|=2$, as wanted.

We will now show that $J H^{*}$ is far from having any diameter 2 property. Our aim is to prove that $B_{J H^{*}}$ has $w^{*}$-slices with arbitrary small diameter. In fact, we will find $x \in S_{J H}$ such that $\inf _{\alpha>0} \operatorname{diam}\left(S\left(B_{J H^{*}}, x, \alpha\right)\right)=0$.

If we denote by

$$
A:=\left\{\sum_{i=1}^{n} \lambda_{i} f_{S_{i}} \mid \begin{array}{c}
\lambda_{i} \in\{-1,1\} \\
\left\{S_{1}, \ldots, S_{n}\right\} \text { is a family of admissible segments in } T
\end{array}\right\}
$$

it is clear that $A \subseteq B_{J H^{*}}$ is a norming subset (by the definition of the norm on $J H)$. Hence

$$
\overline{c o} w^{*}(A)=B_{J H^{*}}
$$

by the Hahn-Banach theorem.

Now we are ready to show that $B_{J H^{*}}$ has $w^{*}$-slices of arbitrarily small diameter.

Theorem 3.3. There exists $x \in S_{J H}$ satisfying that

$$
\inf _{\alpha>0} \operatorname{diam}\left(S\left(B_{J H^{*}}, x, \alpha\right)\right)=0 .
$$


Proof. Pick $0<\varepsilon<\frac{1}{4}$ and put

$$
x=(1-\varepsilon) e_{(0,0)}+\varepsilon e_{(1,0)}-\varepsilon e_{(1,1)}-\varepsilon e_{(2,0)}-\varepsilon e_{(2,1)}-\varepsilon e_{(2,2)}+\varepsilon e_{(2,3)} .
$$

It is clear that $\|x\| \geq 1$, since $\{\{(0,0),(1,0)\}\}$ is a family of admissible segments. It can also be checked that if $\left\{S_{1}, \ldots, S_{r}\right\}$ is a family of admissible segments in $T$ which is different from the family $\{\{(0,0),(1,0)\}\}$ then

$$
\sum_{i=1}^{r}\left|\sum_{t \in S_{i}} x(t)\right| \leq \max \{1-\varepsilon, 4 \varepsilon\}<1 .
$$

Hence $\|x\|=1$. Moreover, if we take $\left\{S_{1}, \ldots, S_{r}\right\}$ to be a family of admissible segments and $\lambda_{1}, \ldots, \lambda_{r} \in\{-1,1\}$ such that

$$
\sum_{i=1}^{r} \lambda_{i} f_{S_{i}}(x)>1-\alpha
$$

for $0<\alpha<\min \{1-4 \varepsilon, \varepsilon\}<1$ then $r=1, S_{1}=\{(0,0),(1,0)\}$ and $\lambda_{1}=1$. So

$$
S(A, x, \alpha)=\left\{f_{\{(0,0),(1,0)\}}\right\} \Rightarrow \inf _{\alpha>0} \operatorname{diam}(S(A, x, \alpha))=0 .
$$

Now Theorem 1.1 applies and as a consequence we get that

$$
\inf _{\alpha>0} \operatorname{diam}\left(S\left(B_{J H^{*}}, x, \alpha\right)\right)=0,
$$

so we are done.

Remark that the element $x$ of Theorem 3.3 is a Fréchet differentiability point of $B_{J H}$, see [4], so as a consequence of the above result we deduce that the unit ball $J H^{*}$ has denting points.

\section{The SPACE $J H_{\infty}$.}

We begin with the construction of $J H_{\infty}$ from $J H$, by a process similar to the construction of $J T_{\infty}$ from $J T$.

We consider $T$ as in section 2. A segment $S=\left\{t_{1}, \ldots, t_{k}\right\}$ is a $n-m$ segment, for $n \leq m$, if $\left|t_{1}\right|=n$ and $\left|t_{k}\right|=m$.

If $\left\{S_{1}, \ldots, S_{k}\right\}$ is a finite family of segments in $T$, we say that is admissible if

(1) There exist natural numbers $n, m$ satisfying $n \leq m$ and $S_{i}$ is a $n-m$ segment for every $i \in\{1, \ldots, k\}$.

(2) $S_{i} \cap S_{j}=\emptyset$ if $i \neq j$.

Given $x: T \longrightarrow \mathbb{R}$ a finitely nonzero function we define

$$
\|x\|:=\sup \sum_{i=1}^{k}\left|\sum_{t \in S_{i}} x(t)\right|,
$$


where the sup is taken over all families of admissible segments $\left\{S_{1}, \ldots, S_{k}\right\}$ of $T$. We define the space $J H_{\infty}$ as the completion of the space of finitely nonzero functions on $T$ in the above norm. If $S \subseteq T$ is a segment, then we denote

$$
f_{S}(x):=\sum_{t \in S} x(t)
$$

Note that $f_{S} \in S_{\left(J H_{\infty}\right)^{*} \text {. }}$

Moreover, in view of the definition of the norm we have that given a family of admissible segments $\left\{S_{1}, \ldots, S_{k}\right\}$ then $\sum_{i=1}^{k} \lambda_{i} f_{S_{i}} \in B_{\left(J H_{\infty}\right)^{*}}$, whenever $\lambda_{1}, \ldots, \lambda_{k} \in\{-1,1\}$.

Given $\alpha \in T$ define

$$
e_{\alpha}(\beta):=\left\{\begin{array}{cc}
1 & \text { if } \beta=\alpha \\
0 & \text { otherwise }
\end{array}\right.
$$

Let us remark that $J H_{\infty}$ is not isomorphic to $J H$. Indeed, we know that $J H$ does not contain isomorphic copies of $\ell_{1}$. However it is enough consider the sequence $\left\{e_{\alpha_{n}}\right\}$, where $\left\{\alpha_{n}\right\}$ is an infinite sequence of immediate successors of the first node in $T$, to get an isometric copy of the usual basis in $\ell_{1}$. Furthermore it is clear that $J H_{\infty}$ contains isometric copies of $J H$.

Now, we can get, as in the previous section, the following result.

Theorem 4.1. $J H_{\infty}$ has the strong diameter two property (and so the norm of $J H_{\infty}^{*}$ is octahedral).

Now we pass to study diameter two properties in $J H_{\infty}^{*}$. To this aim, the next Lemma will help us to estimate the diameter of certain $w^{*}$-slices in $B_{J H_{\infty}^{*}}$.

Proposition 4.2. Let $R, S$ be two disjoint segments in $T$ which are $p-q$ and $p-r$ segments for suitable $p, q, r \in \mathbb{N}, p \leq q \leq r$. Then

$$
\left\|f_{R}-f_{S}\right\| \leq \frac{5}{3}
$$

Proof. If $r=q$, then $\{S, R\}$ is a family of admissible segments in $T$. Hence

$$
\left\|f_{R}-f_{S}\right\|=1<\frac{5}{3}
$$

Now, we assume that $q<r$. Then we can find $U$, a $p-q$ segment, and $V$, a $(q+1)-r$ segment, such that

$$
U \cup V=R \Rightarrow f_{R}=f_{U}+f_{V} .
$$

Let $\alpha \in \mathbb{R}_{0}^{+}$be such that $\left\|f_{R}-f_{S}\right\|=2-\alpha$ and $\varepsilon \in \mathbb{R}^{+}$. Then there exists a finitely nonzero function $x: T \longrightarrow \mathbb{R},\|x\| \leq 1$, such that

$$
\left(f_{R}-f_{S}\right)(x)>2-\alpha-\varepsilon \Rightarrow f_{R}(x)>1-\alpha-\varepsilon \text { and } f_{S}(x)<-1+\alpha+\varepsilon .
$$

As $U$ is a $p-q$ segment disjoint with $S$ we have that $\{U, S\}$ is a family of admissible segments. As a consequence $\left\|f_{U}-f_{S}\right\| \leq 1$. Hence

$$
2-\alpha-\varepsilon<f_{R}(x)-f_{S}(x)=\left(f_{U}-f_{S}\right)(x)+f_{V}(x) \leq 1+f_{V}(x)
$$

and so

$$
f_{V}(x)>1-\alpha-\varepsilon
$$


Moreover

$$
1 \geq f_{R}(x)=f_{U}(x)+f_{V}(x) \geq 1-\alpha-\varepsilon+f_{U}(x)
$$

hence

$$
f_{U}(x) \leq \alpha+\varepsilon
$$

Now, again from the fact that $\{S, U\}$ is a family of admissible segments, we have $\left\|f_{U}+f_{S}\right\| \leq 1$. Hence

$$
-1 \leq\left(f_{U}+f_{S}\right)(x)<f_{U}(x)+(-1+\alpha+\varepsilon) .
$$

Then

$$
f_{U}(x)>-\alpha-\varepsilon .
$$

From (4.1) and (4.2) it follows that

$$
\left|f_{U}(x)\right| \leq \alpha+\varepsilon \text {. }
$$

Now, as $x$ has finite support, we can find $W$, a $(q+1)-r$ segment, such that $S \cup W$ is a $p-r$ segment disjoint with $R$ and we can assume that $x(t)=0 \forall t \in W$. From this we deduce that $\{R, S \cup W\}$ is a family of admissible segments in $T$. Hence

$$
1 \geq\|x\| \geq\left|f_{R}(x)\right|+\left|f_{S \cup W}(x)\right|=\left|f_{U}(x)+f_{V}(x)\right|+\left|f_{S}(x)\right| \geq
$$

$\geq\left|f_{V}(x)\right|-\left|f_{U}(x)\right|+\left|f_{S}(x)\right| \geq(1-\alpha-\varepsilon)-(\alpha+\varepsilon)+(1-\alpha-\varepsilon)=2-3 \alpha-3 \varepsilon$.

From the arbitrariness of $\varepsilon$ we deduce that

$$
1 \geq 2-3 \alpha \Rightarrow \alpha \geq \frac{1}{3}
$$

Then $\left\|f_{S}-f_{T}\right\|=2-\alpha \leq 2-\frac{1}{3}=\frac{5}{3}$, as we wanted.

Now we can conclude that there are $w^{*}$-slices in $B_{J H_{\infty}^{*}}$ with diameter strictly less than two. In fact, we can find $w^{*}$-slices with diameter less than $\frac{5}{3}+\varepsilon$ for every $0<\varepsilon<\frac{1}{3}$.

Theorem 4.3. There exists $x \in S_{J H_{\infty}}$ such that

$$
\inf _{\alpha>0} S\left(B_{J H_{\infty}^{*}}, x, \alpha\right) \leq \frac{5}{3} .
$$

Proof. We define

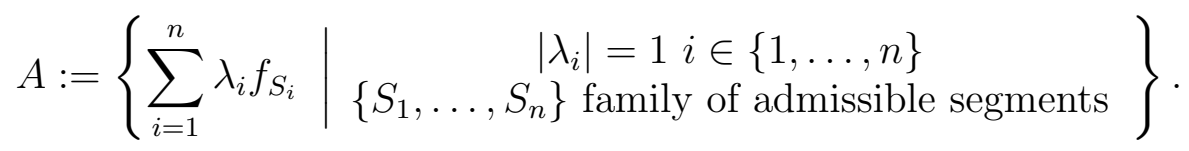

It is clear that $\overline{c o}^{w^{*}}(A)=B_{J H_{\infty}^{*}}$ by an easy separation argument.

Fixed an arbitrary $0<\delta<1 / 2$, we put $x:=(1-\delta) e_{\emptyset}+\delta e_{(1)}$ and pick $0<\alpha<\delta$. Then, if $\sum_{i=1}^{n} \lambda_{i} f_{S_{i}} \in S(A, x, \alpha)$, we have that $n=1, S_{1}$ is a $0-p$ segment for suitable $p \geq 1, \emptyset,(0) \in S_{1}$ and $\lambda_{1}=1$.

So, in order to estimate $\operatorname{diam}(S(A, x, \alpha))$, pick $f_{S}, f_{R} \in S(A, x, \alpha)$. Note that $S \cap R \neq \emptyset$ (both segments contain the set $\{\emptyset,(1)\}$ ). However, we can find two disjoint segments, $U$ and $V$, which are $p-q$ and $p-r$ segments, for suitable $p, q, r \geq 2$, such that

$$
S=(S \cap R) \cup U \text { and } R=(S \cap R) \cup V .
$$


Then

$$
f_{R}-f_{S}=f_{S \cap R}+f_{V}-f_{S \cap R}-f_{U}=f_{V}-f_{U} .
$$

By Proposition 4.2 we deduce that

$$
\left\|f_{V}-f_{U}\right\| \leq \frac{5}{3} \Rightarrow\left\|f_{R}-f_{S}\right\| \leq \frac{5}{3}
$$

From the arbitrariness of $f_{R}, f_{S} \in S(A, x, \alpha)$ we deduce that

$$
\operatorname{diam}(S(A, x, \alpha)) \leq \frac{5}{3}
$$

Hence

$$
\inf _{\alpha>0} \operatorname{diam}(S(A, x, \alpha)) \leq \frac{5}{3} .
$$

Now Theorem 1.1 applies and

$$
\inf _{\alpha>0} \operatorname{diam}\left(S\left(B_{J H_{\infty}^{*}}, x, \alpha\right)\right) \leq \frac{5}{3},
$$

so we are done.

In particular, the above theorem shows that $J H_{\infty}^{*}$ fails the $w^{*}$-slice diameter two property, and so every diameter two property.

In view of the element $x$ defined in last theorem, it seems that $\emptyset \in \operatorname{supp}(x)$ is a very important condition in order to get the desired result (it allowed us to describe easily the elements of $S(A, x, \alpha))$. This will become clear in the next section.

\section{A hyperplane of $J H_{\infty}^{*}$ SATisfying the $w^{*}$-STRong Diameter TWO PROPERTY.}

We will consider $T$ defined as in the previous section. Let

$$
N:=\left\{\begin{array}{l|l}
x: T \longrightarrow \mathbb{R} & \begin{array}{c}
x \text { is a finitely nonzero function } \\
x(\emptyset)=0
\end{array}
\end{array}\right\} .
$$

Now consider on $N$ the norm defined in the previous section. In other words

$$
\|x\|:=\sup \sum_{i=1}^{k}\left|\sum_{t \in S_{i}} x(t)\right|
$$

where the sup is taken over all families of admissible segments $\left\{S_{1}, \ldots, S_{k}\right\}$ in $T$.

Now we define $M$ as the completion of $N$ under the above norm.

Note that $i: N \hookrightarrow J H_{\infty}$ is a linear isometry. So, it can be uniquely extended to a linear isometry $\Phi: M \longrightarrow J H_{\infty}$ and, as a consequence, $M$ can be viewed as a closed subspace of $J H_{\infty}$.

Remark 5.1. Given $x \in N$, note that in the definition of the norm we need only to consider families of admissible segments which are $p-q$ segments with $p \geq 1$. This is an important fact which will allow us to conclude the $w^{*}$-strong diameter two property in $M^{*}$ 
For $S \subseteq T$ a segment, we define $f_{S} \in M^{*}$ by

$$
f_{S}(x)=\sum_{t \in S} x(t) \quad \forall x \in N .
$$

The first consequence of the Remark 5.1 is that

$$
A:=\left\{\begin{array}{c|c}
\sum_{i=1}^{n} \lambda_{i} f_{S_{i}} & \begin{array}{c}
\left|\lambda_{i}\right|=1 \forall i \in\{1, \ldots, n\} \\
\left\{S_{1}, \ldots, S_{n}\right\} \text { family of admissible segments } \\
\emptyset \notin S_{i} \forall i \in\{1, \ldots, n\}
\end{array}
\end{array}\right\}
$$

is a norming set in $B_{M^{*}}$. Hence

$$
B_{M^{*}}=\overline{c o}^{w^{*}}(A),
$$

is an immediate consequence of Hahn-Banach's theorem.

We will use (5.1) in order to prove that $M^{*}$ enjoys the $w^{*}$-strong diameter two property.

Theorem 5.2. $M^{*}$ has the $w^{*}$-strong diameter two property.

Proof. Let $C:=\sum_{i=1}^{n} \lambda_{i} S\left(B_{M^{*}}, x_{i}, \varepsilon\right)$ be a convex combination of $w^{*}$-slices in $B_{M^{*}}$, where $x_{1}, \ldots, x_{n}$ are finitely non-zero functions defined on $T$. Our goal is to prove that $\operatorname{diam}(C)=2$.

To this aim, from (5.1), for each $i \in\{1, \ldots, n\}$ we can find $n_{i} \in \mathbb{N}$, a family of admissible segments in $T,\left\{S_{1}^{i}, \ldots, S_{n_{i}}^{i}\right\}$, and $\mu_{1}^{i}, \ldots, \mu_{n_{i}}^{i} \in\{-1,1\}$ such that

$$
\sum_{i=1}^{n} \lambda_{i} \sum_{j=1}^{n_{i}} \mu_{j}^{i} f_{S_{j}^{i}} \in C
$$

Now, for every $i \in\{1, \ldots, n\}$ we have that $S_{j}^{i}$ is a $p_{i}-q_{i}$ segment for each $j \in\left\{1, \ldots, n_{i}\right\}$. We can assume that $q_{1}=q_{2}=\ldots=q_{n}=r$ and that $r>\max _{1 \leq i \leq n} p_{i}$ because $x_{1}, \ldots, x_{n}$ have finite support and each element on $T$ has infinitely many offsprings.

Again, due to the finiteness of $\operatorname{supp}\left(x_{i}\right)$ for each $i \in\{1, \ldots, n\}$, we can find a branch $B$ in $T$ such that

$$
B \bigcap\left(\bigcup_{i=1}^{n} \operatorname{supp}\left(x_{i}\right)\right)=\emptyset \text {. }
$$

For each $i \in\{1, \ldots, n\}$ we can choose $S_{i} \subseteq B$, a $p_{i}-r$ segment, in $T$. As $S_{i} \cap \operatorname{supp}\left(x_{i}\right)=\emptyset$ and $\left\{S_{1}^{i}, \ldots, S_{n_{i}}^{i}, S_{i}\right\}$ is a family of admissible segments in $T$ we deduce that

$$
\sum_{i=1}^{n} \lambda_{i}\left(\sum_{j=1}^{n_{i}} \mu_{j}^{i} f_{S_{j}^{i}} \pm f_{S_{i}}\right) \in C
$$

Hence

$$
\operatorname{diam}(C) \geq\left\|\sum_{i=1}^{n} \lambda_{i}\left(\sum_{j=1}^{n_{i}} \mu_{j}^{i} f_{S_{j}^{i}}+f_{S_{i}}\right)-\sum_{i=1}^{n} \lambda_{i}\left(\sum_{j=1}^{n_{i}} \mu_{j}^{i} f_{S_{j}^{i}}-f_{S_{i}}\right)\right\|=
$$




$$
=2\left\|\sum_{i=1}^{n} \lambda_{i} f_{S_{i}}\right\|
$$

Let us prove that $\left\|\sum_{i=1}^{n} \lambda_{i} f_{S_{i}}\right\|=1$. Note that the equality $\left\|\sum_{i=1}^{n} \lambda_{i} f_{S_{i}}\right\| \leq 1$ is clear from the triangle inequality. Moreover, as $S_{i}$ is a $p_{i}-r$ segment in $T$ and $p_{i}<r \forall i \in\{1, \ldots, n\}$, we deduce the existence of $\alpha \in \bigcap_{i=1}^{n} S_{i}$. Now $e_{\alpha} \in S_{M}$. Hence

$$
\left\|\sum_{i=1}^{n} \lambda_{i} f_{S_{i}}\right\| \geq \sum_{i=1}^{n} \lambda_{i} f_{S_{i}}\left(e_{\alpha}\right)=\sum_{i=1}^{n} \lambda_{i}=1 .
$$

Thus $\operatorname{diam}(C)=2$, as desired.

The last theorem shows that $M^{*}$ has the $w^{*}$-strong diameter two property and so the norm of $M$ is octahedral. Moreover, it is easy to check that $M$ has the strong diameter two property, as proved for $J H$, and so the norm of $M^{*}$ is also octahedral. As $M$ is separable, we deduce from the comments in the introduction the following

Corollary 5.3. $M$ has the almost Daugavet property.

Acknowledgement. The authors want to thank an anonymous referee for helping us to improve the final version or this work.

The first author was partially supported by MEC (Spain) Grant MTM201123843 and Junta de Andalucía grants FQM-0199, FQM-1215. The second author was partially supported by MEC (Spain) Grant MTM2012-31755 and Junta de Andalucía Grant FQM-185.

\section{REFERENCES}

1. J. Becerra Guerrero, G. López-Pérez and A. Rodríguez-Palacios, Relatively weakly open sets in closed balls of $C^{*}$-algebras, J. London Math. Soc. 68 (2003), 753-761.

2. J. Becerra Guerrero, G. López-Pérez and A. Rueda Zoca, Octahedral norms and convex combination of slices in Banach spaces, J. Funct. Anal. 266 (4) (2014), 2424-2436.

3. J. Becerra Guerrero and Á. Rodríguez Palacios, Locally uniformly rotund points in convextransitive Banach spaces, J. Math. Anal. Appl. 360 (2009), 108-118.

4. R. Deville, G. Godefroy and V. Zizler, Smoothness and renormings in Banach spaces. Pitman Monographs and Surveys in Pure and Applied Math. 64, 1993.

5. H. Fetter, B. Gamboa de Buen, The James Forest, London Mathematical Society Lecture Notes Series, 236, Cambridge University Press. Cambridge 1997.

6. N. Ghoussoub and B. Maurey, $G_{\delta}$-embeddings in Hilbert space, J. Func. Anal. 61 (1) (1985), 72-97.

7. J. Hagler, A counterexample to several questions about Banach spaces, Studia Math. 60 (4) (1977), 289-308.

8. V. Kadets, V. Shepetska, D. Werner, Thickness of the unit spphere, $\ell_{1}$-types and the almost Daugavet property. Houston J. Math. 37 (3) (2011), 867-878.

9. G. López-Pérez, The big slice phenomena in M-embedded and L-embedded spaces, Proc. Amer. Math. Soc. 134 (2005), 273-282.

10. R.C. James, Bases and Reflexivity of Banach spaces, Ann. Math. 52 (2) (1950), 518-527.

11. R.C. James, A non-reflexive Banach space isometric with its second conjugate space, Proc. of the Nat. Acad. of U.S.A, 37 (3) (1951), 174-177. 
12. R.C. James, A separable somewhat reflexive Banach space with nonseparable dual, Bull. Amer. Math. Soc. 80 (4) (1974), 738-743.

13. J. Lindestrauss and L. Tzafriri, Classical Banach spaces, Springer Verlag. Berlin 1977.

14. O. Nygaard and D. Werner, Slices in the unit ball of a uniform algebra, Arch. Math. 76 (2001), 441-444.

15. W. Schachermayer, A. Sersouri and E. Werner, Moduli of nondentability and the RadonNikodým property in Banach spaces, Israel J. Math. 65 (3) (1989), 225-257.

16. R.V. Shvydkoy, Geometric aspects of the Daugavet property, J. Funct. Anal. 176 (2000), $198-212$.

Departmento de Análisis Matemático, Universidad de Granada, 18071 Granada, SPAIN.

E-mail address: juliobg@ugr.es

E-mail address: glopezp@ugr.es

E-mail address: arz0001@correo.ugr.es 